\title{
Traumatic acute posterior fossa subdural hematoma - A case report and review of literature
}

\author{
Manish Jaiswal, I. Vijay Sundar, Ashok Gandhi, Devendra Purohit, \\ R.S. Mittal \\ Department of Neurosurgery, SMS Medical College and Hospital JLN Marg, Jaipur (302004), \\ Rajasthan, India
}

\begin{abstract}
Traumatic subdural hematomas of the posterior fossa are rare but dangerous neurosurgical emergencies that require prompt diagnosis and management to avoid the uniformly poor outcome. We present a case of a teenager with severe TBI and acute subdural hematoma of the posterior fossa that deteriorated rapidly before surgery but eventually made a good recovery. We also the review the literature concerning traumatic posterior fossa subdural hematomas [PFSDH].
\end{abstract}

Key words: Acute Subdural Hematoma; Posterior Fossa SDH; Head injury.

\section{Introduction}

Acute PFSDHs are rare manifestations of traumatic brain injury with a reported incidence of $0.21 \%$ to $0.27 \%[1,2]$. They are classical neurosurgical emergencies as they can cause abrupt deterioration and death from brainstem compression.

\section{Case report}

A 16 year old male presented to the emergency department with history of unconsciousness following road traffic accident. On examination patient was comatose with Glasgow Coma score (GCS) of E1V2M4. Pupils were bilaterally $4 \mathrm{~mm}$ and sluggishly reactive. Patient was intubated and an immediate Computed tomography scan (CT scan) brain was performed which showed hyper-dense blood in posterior fossa region between cerebellar hemispheres with maximum thickness more than $1 \mathrm{~cm}$ causing pressure on fourth ventricle (Figure 1). Patient was immediately shifted to Operation theatre where posterior fossa craniectomy and foramen magnum decompression was done. Dura was tense and bluish (Figure 2). Durotomy and evacuation of Acute SDH was done. To accommodate cerebral edema dura was left open. Patient was shifted to neurosurgical ICU and kept under intensive monitoring and elective ventilation. He gradually recovered over the next 48 hours and was extubated on the third day. Patient was eventually discharged at 10th post-operative day at which time he was conscious. At last follow up of two months from surgery patient is doing well and is ambulatory without support. 


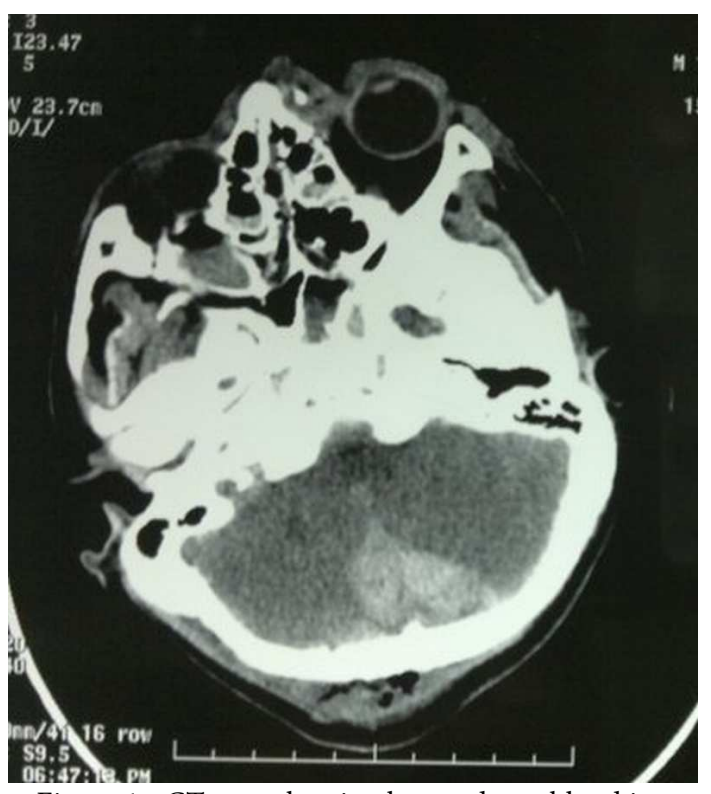

Figure 1 - CT scan showing hyper-dense blood in posterior fossa region causing pressure on fourth ventricle

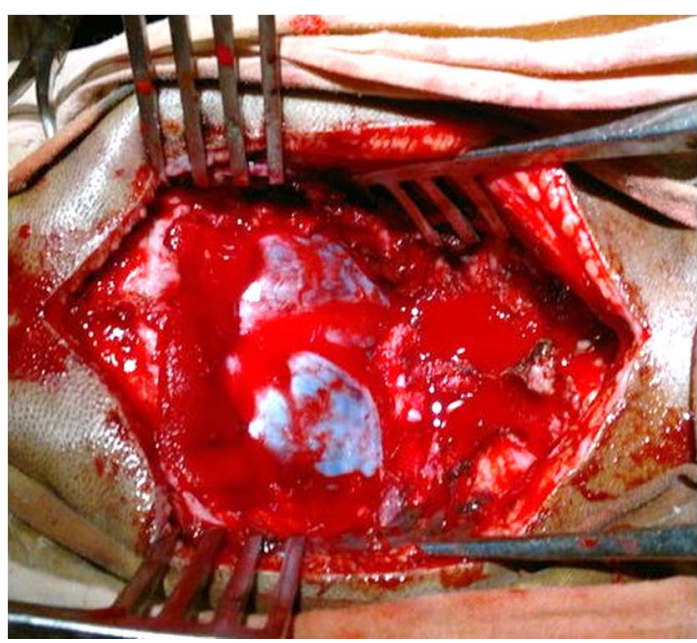

Figure 2 - Posterior fossa craniectomy and foramen magnum decompression showing bluish and tense duramater

\section{Discussion}

PFSDHs constitute the rarest type of posterior fossa traumatic hematomas, accounting for approximately $10 \%$ of posterior fossa traumatic lesions and is roughly 0.3 to $0.8 \%$ of supra-tentorial subdural hematomas $(1,2,3)$.

D'Avella et al (1) classified patients with posterior fossa subdural hematomas into two groups. 1: Conscious patients with hematomas not more than $1 \mathrm{~cm}$ with no signs of pressure effects on the brainstem, fourth ventricle or basal cisterns and 2): patients with hematomas larger than $1 \mathrm{~cm}$, signs of mass effect on the brainstem, fourth ventricle or basal cisterns. Urgent surgery was recommended in the latter group.

Clinical features are diverse and unpredictable ranging from coma or significant depression of consciousness [4] to almost conscious patients. The clinical course during the initial $24-48$ hours is more important $(1,4)$. Progressive or sudden clinical deterioration has been observed frequently within the first several hours after injury.

Typical CT finding of PFSDH is the presence of high attenuation extra-cerebellar/ retrocerebellar collection in a concave-convex shape (1). Occipital fracture, hydrocephalus and subarachnoid hemorrhage could be seen in these patients $(1,4,5)$. Impact in the region of posterior fossa frequently produces contrecoup lesions supratentorially in the frontal and/ or temporal region which are common findings in traumatic PFSDH [6]. Delayed or evolving hemorrhagic lesions of the posterior 
DOI: 10.2478/romneu-2014-0066

fossa have been reported (7) thus emphasizing the need for close monitoring which may include daily CT scans until the lesion stabilizes.

CT scan plays an even more important role in the absence of clinical deterioration. CT parameters such as thickness of hematoma, appearance of the cisterns surrounding the brainstem, and coexisting posterior fossa lesions aid decision making $(1,8)$. The thickness of hematoma, although reflecting only in part the course of the disease, is an important parameter since large hematomas generally need prompt evacuation, whatever the clinical status of the patient. Possible brainstem damage which may co-exist with PFSDH may be responsible for the discrepancy that is sometimes noted between the thickness of the hematoma and the clinical condition $(5,6)$.

Management of PFSDH is individualized rather than algorithmic. The general concept is that an acute posterior fossa subdural hematoma is a neurosurgical emergency as it may cause brainstem compression and acute fourth ventricle obstruction, worsening clinical features and death.

D'Avella et al performed a clinicoradiological analysis of 24 patients of traumatic PFSDH of which 19 were operated upon [1]. They found a high percentage of favorable outcome in the group with initial GCS $>$, or $=8$ whereas a uniformly poor outcome was noted in the group with initial GCS $<8$. They found that only GCS score had independent prognostic significance.

Park et al published their experience of six operated cases of PFSDH and noted that of the four patients with initial GCS of 13 or above three patients deteriorated within 24 hours from trauma.

Patients with thick hematomas and deteriorating clinical status need urgent surgery as do patients with the radiological signs of pressure effects on the fourth ventricle or brain stem or the surrounding cisterns, even when the patient has no neurological deficits (4). Conservative approach may be advocated in PFSDHs which are less than or equal to $1 \mathrm{~cm}$ in thickness with no pressure effect on the brain stem and fourth ventricle and no signs of neurological deficit (9). The rest need close neurosurgical monitoring because of the danger of delayed increase in hematoma or fall in conscious level.

The standard surgical treatment consists of evacuation of the hematoma by suboccipital craniectomy and durotomy (4). The most important prognostic factor in PFSDH is the clinical condition of the patient at the time of surgery $(4,9,10)$. Patients with a Glasgow coma scale score less than 8 have a high percentage of bad outcome and patients with a Glasgow coma scale of 8 or more have better chance of recovery (1).

Early CT scanning is very important as it not only provides data for immediate decision making but also serves as a control to evaluate the progression of the hematoma within the first 24 hours in conservatively managed patients (1). Early diagnosis before neurological deterioration develops and a prompt surgical evacuation of hematoma lead to satisfactory outcome in the treatment of PFSDH patients. 


\section{Conclusion}

PFSDH is a relatively rare manifestation of traumatic brain injury which can have various presentations. The possibility of a progressive course of the lesion or a delayed hematoma should be kept in mind even if initial CT is normal. The most important factor determining the prognosis is level of consciousness just before surgery. Early diagnosis by an early CT scan and a prompt surgical evacuation lead to excellent recovery in patients with PFSDH. We recommend urgent posterior fossa craniectomy and durotomy if the patient has poor initial GCS, deterioration in GCS during hospital stay, initial CT scan showing pressure on fourth ventricle or hematoma more than $1 \mathrm{~cm}$ thick. During surgery it is advisable to accommodate cerebral edema by leaving the dura open or by duraplasty. In all cases pre and post-operative intensive neurosurgical monitoring is mandatory.

\section{Correspondence}

Manish Jaiswal

A 33/48 A 2, Omkareshwar, Varanasi, 221001,

Uttar Pradesh, INDIA

Phone - 091-9799980494

E-mail-manishmlnmc@gmail.com

\section{References}

1. d'Avella D, Servadei F, Scerrati M, Tomei G, Brambilla G, Massaro F, et al: Traumatic acute subdural hematomas of the posterior fossa: clinicoradiological analysis of 24 patients. Acta Neurochir (Wien) 145:1037-1044, 2003

2. Karasawa H, Furuya H, Naito H, Sugiyama K, Ueno J, Kin H: Acute hydrocephalus in posterior fossa injury. J Neurosurg 86: 629-632, 1997

3. Dirim BV, Oruk C, Erdogan N, Gelal F, Uluc E: Traumatic posterior fossa hematomas. Diagn Interv Radiol 11: 14-18, 2005

4. Hecimovic I, Blagus G, Kristek B, Rukovanjski M, Vrankovic D: Successful treatmentof traumatic acute posterior fossa subdural hematoma: report of two cases. Surg Neurol 51: 247-251, 1999

5. Kim YD, Park HK, Chang JC, Cho SJ, Choi SK, Byun PJ: Traumatic Intracerebellar Hematomas. J Korean Neurosurg Soc 37: 213-216, 2005

6. Vrankovic D, Splavski B, Hecimovic I, Kristek B, Dmitrovic B, Rukovanjski M, et al: Anatomical cerebellar protection of contrecoup hematoma development. Analysis of the mechanism of 30 posterior fossa coup hematomas. Neurosurg Rev 23: 156-160, 2000

7. Servadei F, Nanni A, Nasi MT, Zappi D, Vergoni G, Giuliani G, et al: Evolving brain lesions in the first 12 hours after head injury: Analysis of 37 comatose patients. Neurosurgery 37: 899-907, 1995

8. Servadei F: Prognostic factors in severely head injured patients with subdural haematoma's. Acta Neurochir (Wien) 139: 273-278, 1997

9. Ashkenazi E, Carmon M, Pasternak D, Israel Z, Beni L, Pomeranz S: Conservative treatment of a traumatic subdural hematoma of the posterior fossa in a child: case report. J Trauma 36: 406-407, 1994

10. Motohashi O, Komeyama M, Shimosegawa Y, Fujimori K, Sugai K, Onuma T: Single burr hole evacuation for traumatic acute subdural hematoma of the posterior fossa in the emergency room. J Neuro-trauma 19: 993-998, 2002 